\title{
DELECIÓN 17p11.2 EN UNA NIÑA DISMÓRFICA CON EVIDENCIA FENOTÍPICA DE SÍNDROME DE SMITH-MAGENIS Y UNA REVISIÓN DE LA LITERATURA
}

\begin{abstract}
Sergio Talavera Vargas Machuca ${ }^{1}$, Ismenia Gamboa Oré2, Ruth Barrientos Marca ${ }^{3}$, Dina Torres Gonzales ${ }^{4}$, Jackeline Zevallos Murgado ${ }^{5}$, Leonor Contreras Aguilar ${ }^{5}$, María Luisa Fajardo Loo ${ }^{6}$
\end{abstract}

\begin{abstract}
RESUMEN
Las deleciones cromosómicas son anomalías estructurales que originan pérdidas del material genómico y dependiendo de su extensión suelen dar lugar a condiciones genéticas irreversibles muy discapacitantes. La deleción intersticial del brazo corto del cromosoma 17 en la región 17p11.2 está relacionada a la aparición de características fenotípicas comunes a una condición genética conocida como Síndrome de Smith-Magenis. La deleción, en un rango que varía entre $<1.5 \mathrm{Mb}$ y $9 \mathrm{Mb}$ se manifiesta con dismorfias craneofaciales, retardo en el desarrollo psicomotor, problemas del habla, trastornos del sueño, anomalías de las extremidades, además de malformaciones cerebrales, cardiacas y renales. Reportamos el caso de una niña de 10 meses, con braquicefalia, frente abombada, ojos hundidos, pliegues epicánticos, orejas malformadas, philtrum corto, boca pequeña, laringomalacia, trastornos del sueño y ventriculomegalia. El cariotipo de la niña fue 46,XX, del (17) (p11.2), no se realizaron pruebas moleculares por no estar disponibles, pero por las evidencias fenotípicas, los aspectos clínicos y citogenéticos clásicos, se propone un cuadro de Síndrome de Smith-Magenis. Sugerimos el seguimiento del caso y ejecución de pruebas moleculares modernas para un diagnóstico definitivo.
\end{abstract}

Palabras Clave: Cromosoma 17; Deleción intersticial 17p; Síndrome de deleción 17p11.2; Región cromosómica Smith-Magenis; Síndrome de Smith-Magenis(Fuente: DeCS BIREME).

\section{7p11.2 DELETION IN A DYSMORPHIC GIRL WITH PHENOTIPIC EVIDENCE OF SMITH-MAGENIS SYNDROME AND A LITERATURE REVIEW}

\begin{abstract}
Chromosomal deletions are structural abnormalities that cause loss of genomic material, depending on its length they use to generate several disabling genetic conditions. Interstitial deletion of chromosome 17 short arm in 17p11.2 region is related to the appearance of phenotypic characteristics related to a genetic condition known as Smith-Magenis syndrome. This deletions spans in a range between $<1.5$ and $9 \mathrm{Mb}$, shows some features as craniofacial dysmorphia, delayed psychomotor development, speech problems, sleep disorders, limb abnormalities, malformations of brain, heart and kidney also are expressed. We present a case of a 10 monthsold girl with brachycephaly, bulging forehead, sunken eyes, epicanthal folds, malformed ears, short philtrum, small mouth and ventriculomegaly. The gir'sl karyotype was 46,XX,del(17)(p11.2), no molecular tests were performed because they were not available, but the phenotypic evidence, the clinical and classical cytogenetic aspects are suggestives of Smith-Magenis Syndrome. We propose to follow up of this case and perform modern molecular tests for a definitive diagnosis.
\end{abstract}

Key Words: chromosome 17; 17p interstitial deletion; Chromosome 17p11.2 deletion síndrome; Smith-Magenis chromosomic región; Smith-Magenis síndrome (Source:MeSH NLM).

\footnotetext{
Biólogo. Docente de la Facultad de Medicina de la Universidad San Martín de Porres y de Ciencias de la Salud de la Universidad Norbert Wiener Laboratorio de Citogenética del Instituto Nacional Materno Perinatal Lima Perú.

2 Bióloga. Docente de la Facultad de Medicina de la Universidad Nacional Federico Villarreal y San Martín de Porres. Laboratorio de Citogenética del Instituto Nacional Materno Perinatal. Lima Perú.

3 Bióloga. Docente de la Facultad de Enfermería Universidad Peruana Cayetano Heredia. Laboratorio de Citogenética del Instituto Nacional Materno Perinatal. Lima Perú.

Bióloga. Docente de la Facultad de Medicina de la Universidad de San Martín de Porres.

Bióloga. Laboratorio de Citogenética, Instituto Nacional Materno Perinatal.

Médico Genetista. Instituto Nacional Materno Perinatal. Lima, Perú.
}

Recibido: 20-12-16 Aprobado: 30-12-16 


\section{INTRODUCCIÓN}

Las deleciones son anomalías estructurales que ocurren por quiebres en ciertas regiones cromosómicas con pérdidas de material genético y suelen vincularse a dismorfias y anomalías graves del desarrollo. Estas pérdidas pueden ocurrir por dos quiebres (deleción intersticial) o por uno solo (deleción terminal) en uno de los brazos del cromosoma ${ }^{1}$. La región delecionada oscila entre $<1.5 \mathrm{Mb}$ hasta $9 \mathrm{Mb}$ y la mayoría de pacientes tienen una deleción de $\sim 5 \mathrm{Mb}(\sim 10-11 \% \text { del cromosoma } 17)^{2,3}$. El fenotipo puede variar dependiendo de la extensión del segmento delecionado, no obstante se han descrito algunas características más o menos constantes que catalogan a estas condiciones como un síndrome. Las deleciones intersticiales del cromosoma 17 han sido vinculadas a ciertas condiciones como el síndrome de Miller-Dieker que se caracteriza por lisencefalia tipo I, microcefalia, frente prominente, estrechamiento craneal bitemporal, nariz estrecha con narinas antevertidas, labio superior prominente y micrognatia, ocasionada por mutaciones en el cromosoma por deleción 17p13, y que se manifiesta con retraso psicomotor severo, epilepsia de difícil control y trastornos de la alimentación ${ }^{4}$. Otra condición descrita es el Síndrome de SmithMagenis vinculada a deleción intersticial 17 p11.2 cuyas características clínicas incluyen braquicefalia, hipoplasia medio facial, prognatismo, voz ronca, retardo en el habla con o sin pérdida de audición, retardo psicomotor y del crecimiento patrones anormales del sueño y problemas de comportamiento 5 . Describimos el caso de una niña nacida a las 33 semanas de edad gestacional con retardo en el crecimiento, dismorfias craneofaciales, anomalías de las extremidades, trastorno del sueño y con cariotipo $46, X X$, del (17)(p11.2) sin estudios confirmativos por citogenética molecular de deleción intersticial y de quien sugerimos podría tratarse de un cuadro clínico compatible con Sindrome de Smith-Magenis.

\section{REPORTE DEL CASO}

La paciente, una niña de 10 meses de edad nacida por cesárea de una madre de 20 años, a las 33 semanas de edad gestacional, con un peso de $1576 \mathrm{~g}$, talla: $38 \mathrm{~cm}$, perímetro cefálico: $29.5 \mathrm{~cm}$, perímetro torácico: $26 \mathrm{~cm}$. El puntaje de Apgar fue de 8 al primer minuto y a los 5 minutos. Al examen físico presentó una facies dismórfica atípica con frente abombada, ojos hundidos, cejas ralas, puente nasal deprimido, con orejas de implantación baja, suturas cabalgadas, abdomen globuloso. El informe de neuropediatría revela en la ecografía transfontanelar una ventriculomegalia mínima sin hemorragia intraventricular. Los miembros superiores flexionados con hiperextensión, línea simiana. (Figura 1) El tercer dedo del pie izquierdo dorsiflexo cabalgando al cuarto. El informe cardiológico revela una persistencia del conducto arterioso de $1 \mathrm{~mm}$ y comunicación interventricular perimembranosa de $2 \mathrm{~mm}$. Soplo sistólico II/VI. Tono muscular levemente disminuido.

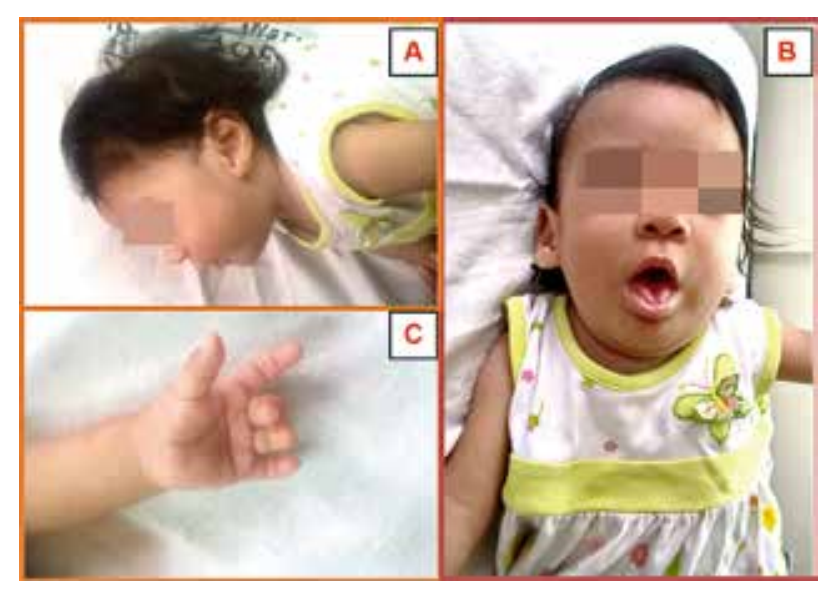

Figura 1. Algunos rasgos fenotípicos de la paciente. En A: Frente abombada, orejas de implantación baja, puente nasal deprimido, ojos hundidos. B: Cejas ralas, pliegues epicánticos, philtrum corto, boca pequeña y revertida hacia abajo. C: Línea simiana izquierda.

La evaluación oftalmológica mostró condiciones normales. El informe ecográfico renal también concluye una constitución normal a nivel renal. Además de estos hallazgos la niña presentó laringomalacia y ritmos alterados del sueño.

Solicitado el estudio cromosómico de la paciente se realiza el cultivo de linfocitos en medio de cultivo RPMI 1640 obteniéndose cromosomas en metafase a las 72 horas de cultivada la sangre. Las láminas preparadas fueron sometidas a tratamiento enzimático con tripsina bovina 1:250 para la obtención de bandas GTG a una resolución de 550 bandas, las lecturas se efectuaron con un microscopio óptico binocular marca Carl Zeiss Modelo Jenamed, encontrándose un cariotipo constante de 46,XX, del (17)(p11.2) en todas las células analizadas (Figuras 2). El cariotipo de la madre del propositus fue de constitución normal 46,XX. Lamentablemente, no se pudo realizar cariotipo al padre del propositus, que hubiera ayudado a esclarecer el origen de la cromosomopatía. Se sugiere el empleo de técnicas moleculares más modernas como CGH-array o MLPA para determinar la extensión del segmento delecionado y afinar de esa manera el diagnóstico definitivo que ayude a mejorar la calidad de vida de la paciente. 

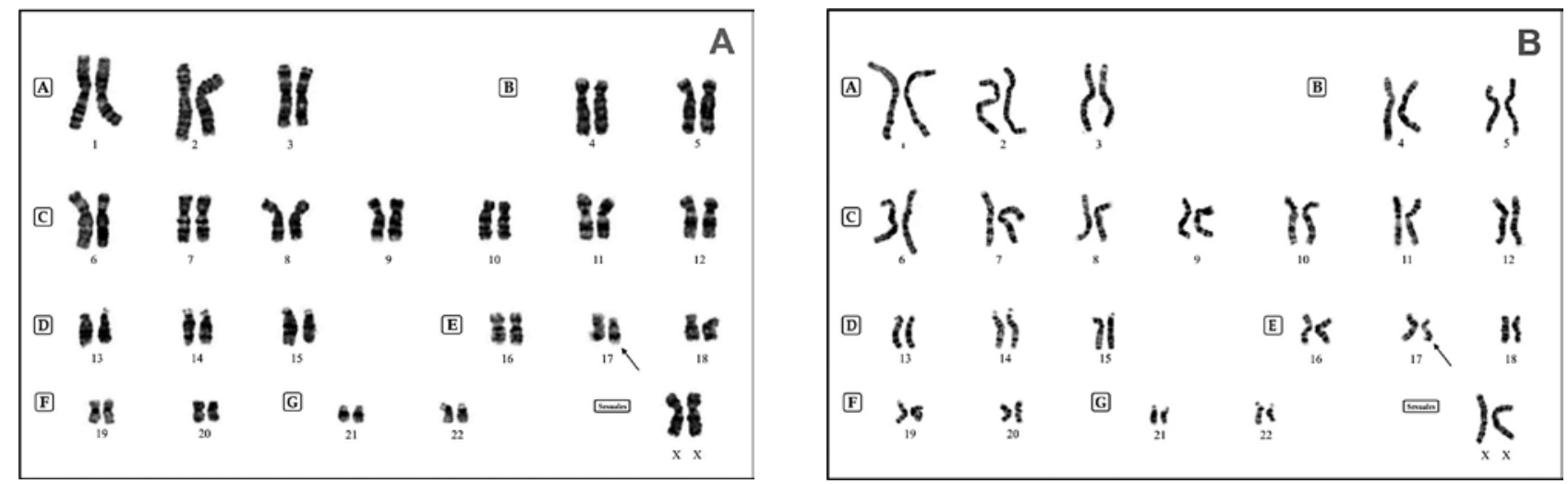

Figura 2. Cariogramas de la paciente a dos diferentes resoluciones de bandas GTG. En A: 450 bandas. B: 650 bandas. En ambas fotografías las flechas señalan al cromosoma del par 17 con el segmento delecionado en $17 p 11.2$

\section{DISCUSIÓN}

Son pocas las condiciones genéticas relacionadas a pérdidas parciales del brazo corto del cromosoma 17, estas son conocidas en general como deleciones parciales del cromosoma 17p o monosomías parciales de 17p. Esta grave y discapacitante afección con pobre desarrollo psicomotor de los individuos que la padecen, suele presentarse como una condición de novo y rara vez heredada de padres portadores de reordenamientos cromosómicos balanceados que comprometan al cromosoma 17.

En el grupo de trastornos hematológicos también se plantea una relación de las deleciones $17 \mathrm{p}$ - con síndromes mielodisplásicos caracterizados por una disgranulopoiesis "típica"6

Patil y Bartley en 1984 reportan el caso de una niña de 4 años que presentaba una deleción intersticial del cromosoma 17p11.2. Ella tenía un cuadro de retardo mental, hipotonía, problemas de lenguaje, orejas pequeñas, sordera, esotropia, displasia del esmalte dental y una premaxila prominente ${ }^{7}$.

Smith et al. En 1986 describen detalladamente el fenotipo asociado a la deleción 17 p11.2 en 9 pacientes no relacionados entre ellos, las características comunes son braquicefalia, hipoplasia facial, prognatismo, problemas de lenguaje, retardo en el desarrollo psicomotor y alteraciones de la conducta. Menciona además la intensificación del cuadro clínico en un caso de deleción completa 17p11.2, con paladar hendido y anomalías severas cardiacas, esqueléticas y genitourinarias ${ }^{5}$.

El síndrome de Smith-Magenis es una condición genética rara pues su prevalencia se estima 1/25000 y $90 \%$ de los casos se debe a una deleción intersticial del cromosoma 17 en $17 p 11.2^{8}$. El síndrome fue descrito por primera vez por Ana Smith y Ellen Magenis a principios de los 80 de ahí deriva el nombre de esta condición ${ }^{5,9}$ y está caracterizado, además del patrón cráneo facial de anomalías ya descrito, por problemas de desarrollo y del comportamiento, conducta autoagresiva, desórdenes del sueño y autismo ${ }^{10,11}$

Greenberg et al en 1991 sugieren un posible vínculo entre pacientes afectados de Síndrome de Smith-Magenis con una neuropatía periférica en la forma CMT1A de la enfermedad de Charcot-Marie-Tooth, sin embargo concluyeron que ningún paciente con CMT1A mostró signos de Síndrome de Smith-Magenis ${ }^{2}$.

Ese mismo año Moncla et al describen tres casos nuevos de Síndrome de Smith-Magenis con una descripción fenotípica de las anomalías craneofaciales, características del síndrome, muy bien documentados de manera gráfica ${ }^{12}$.

En un gran estudio multidisciplinario en 27 pacientes con Síndrome de Smith-Magenis se reconoce nuevos hallazgos comunes a los pacientes afectados como son las anomalías otolaringológicas, oculares, y trastornos del sueño (especialmente disminución de REM (Movimientos Rápidos de los Ojos durante el sueño), afecciones auditivas, escoliosis, anomalías cerebrales, especialmente ventriculomegalia, anomalías cardiacas y renales, bajos niveles de tiroxina y de inmunoglobulina. Retardo mental en rango moderado ${ }^{13}$.

Se ha relacionado los trastornos del sueño en pacientes con Síndrome de Smith-Magenis principalmente por presentar ritmos invertidos de secreción de melatonina. Un tratamiento con melatonina y antagonistas beta adrenérgicos minimiza notablemente los disturbios del sueño ${ }^{14}$. De Leersnyder et al., en el año 2001 documentan trastornos del sueño en 20 niños con Síndrome de Smith-Magenis con ritmo circadiano invertido de melatonina plasmática, melatonina urinaria y 6-sulfatoximelatonina urinaria en 8 de ellos. Los autores sugieren que una haploinsuficiencia en COP53 es la responsable de la perturbación ${ }^{15}$.

Smith et al. en 2002 estudiaron perfiles lipídicos en 49 niños con Síndrome de Smith-Magenis e hicieron la observación de los valores para colesterol sérico total, triglicéridos, colesterol LDL y colesterol HDL y compararon 
sus resultados con los controles normales. El colesterol total medio fue significativamente más elevado que en el grupo control ${ }^{16}$.

Una deleción intersticial tan pequeña como es la 17p11.2 requiere de pruebas citogenéticas de cariotipo en alta resolución y uso de modernas técnicas moleculares. Moncla en 1993 mapea 3 microdeleciones en pacientes con Síndrome de Smith-Magenis, mediante análisis de Southern blot, demostrando que los sujetos en estudio presentaban una deleción para los marcadores D17S29 y D17S71. Los autores sugieren que una región inestable localizada entre la región del Síndrome de Smith-Magenis y la región CMT1A podría ser un punto de acceso para los reordenamientos que conduzcan a microdeleciones en el Síndrome de Smith-Magenis ${ }^{17}$.

El uso de hibridación in situ fluorescente (FISH) para diagnóstico de Síndrome de Smith Magenis por Juyal et al en 1995 demostró una deleción 17p11.2, la misma que había sido indetectable tanto por métodos de cuantificación de ADN cromosómico por citometría de flujo como por citogenética convencional ${ }^{18}$.

Chevillard et al. 1993 identifican la primera secuencia expresada, localizada en la región crítica del Síndrome de Smith-Magenis, el gen que codifica RNA U3 nuclear ${ }^{19}$. En 1996 Koyama et al.localizan el homólogo humano (LLGL1) del gen Llglh de ratón.. Este gen fue mapeado por hibridación in situ fluorescente (FISH) en el cromosoma 17p11.2 En los pacientes con Síndrome de Smith-Magenis esta región está delecionada. En cuatro pacientes, la sonda para LLGL1 falló en hibridizar con uno de los cromosomas 17 homólogos sugiriendo que el gen puede jugar un papel principal en la patogenia del Síndrome de Smith-Magenis ${ }^{20}$.

Lucas et al (2001) plantean que ciertas características del Síndrome de Smith-Magenis como la hipotonía, el retardo mental y los trastornos del comportamiento podrían ser efecto de un exceso de sustrato dTTP y de mitocondrias defectuosas $^{21}$.

En un estudio de tres individuos con características clínicas de Síndrome de Smith-Magenis pero sin deleciones 17 p11.2 detectables por técnicas de hibridación in situ fluorescente, realizado por Slager et al. (2003) encuentran en dos de ellos una deleción de una sola citosina en el gen RAI1, sosteniendo que la haploinsuficiencia de RAI1 probablemente sea responsable de los aspectos conductuales, neurológicos, otolaringológicos y craneofaciales del Síndrome de Smith-Magenis ${ }^{22}$.

En otro estudio caso control de Arrays citogenómicos efectuado en el año 2011 por Kaminsky et al., focalizado en 14 regiones variantes en número de copias que incluía entre otras anomalías cromosómicas estructurales a la deleción $17 \mathrm{p} 11.2$, se identificaron 16 casos con una frecuencia de 1 en $984^{23}$.
En este reporte presentamos el caso de una niña de 10 meses de nacida con facies dismórfica atípica con frente abombada, suturas cabalgadas ojos hundidos y de orientación mongoloide, pliegues epicanticos, cejas ralas, puente nasal deprimido, nariz pequeña y respingada, narinas antevertidas, orejas grandes de implantación baja y posterior, philtrum corto, boca pequeña, protrusión de lengua y laringomalacia El abdomen es globuloso. Por ecografía transfontanelar se revela ventriculomegalia. Hipotonía muscular, un retardo en el desarrollo psicomotor y trastornos del sueño. No se identifican cardiopatías congénitas severas ni malformaciones renales. Todas estas características coinciden con las anteriormente descritas y son sugerentes de que la niña podría estar afectada del Síndrome de Smith-Magenis. Dada la temprana edad de la paciente se recomienda el seguimiento por diferentes especialidades como endocrinología, estomatología, neurología, cardiología, terapia física y rehabilitación ya que algunas características fenotípicas como el retardo mental o ciertos rasgos faciales como el prognatismo se van manifestando o acrecientan con la edad.

\section{SUGERENCIAS}

Es esta la primera vez que se presenta una deleción del brazo corto del cromosoma 17 en nuestra institución y lamentablemente en el Laboratorio de Citogenética del Instituto Nacional Materno Perinatal no contamos con la tecnología adecuada que permita afinar la presunción diagnóstica, como sondas fluorescentes que identifiquen la presencia o ausencia de la región Smith Magenis para Hibridación in situ Fluorescente (FISH), métodos más modernos y precisos para la detección de pacientes con Síndrome de Smith-Magenis comprenden técnicas como el Array-CGH o hibridación genómica comparativa, tecnología con la cual tampoco contamos en nuestra institución. Sugerimos para la culminación de este caso realizar las pruebas moleculares indicadas en algún laboratorio nacional o extranjero que cuente con estos recursos. Al margen de la identificación o descarte de la condición como síndrome de Smith Magenis por métodos moleculares, se sugiere brindar la adecuada consejería genética y el manejo integral de la paciente y sus familiares por las diferentes especialidades de la institución.

\section{REFERENCIAS BIBLIOGRÁFICAS}

1. Griffiths AJF, Miller JH, Suzuki DT, et al An Introduction to Genetic Analysis. 7th edition. New York: W. H. Freeman; 2000.

2. Greenberg, F., Guzzetta, V., Montes de Oca-Luna, R., Magenis, R. E., Smith, A. C. M., Richter, S. F., Kondo, I., Dobyns, W. B., Patel, P. I., Lupski, J. R. Molecular analysis of the Smith-Magenis syndrome: a possible contiguous-gene syndrome associated with del(17)(p11.2). Am. J. Hum. Genet. 49: 1207-1218, 1991.

3. Trask BJ, Mefford H, van den EG, Massa HF, Juyal RC, Potocki L, Finucane B, Abuelo DN, Witt DR, Magenis E, Baldini A, Greenberg F, Lupski JR, Patel PI. Quantification 
by flow cytometry of chromosome-17 deletions in SmithMagenis syndrome patients. Hum Genet 1996;98:710-18.

4. O. López Suárez, C. Curros Novo, A. Ansede López, F. Claro González, E. Rodrigo Sáez, M. Castro-Gago Lisencefalia tipo I con microdeleción 17p13.3: síndrome de Miller-Dieker Acta Pediatr Esp. 2006; 64(10): 506-509

5. Smith, A. C. M., McGavran, L., Robinson, J., Waldstein, G., Macfarlane, J., Zonana, J., Reiss, J., Lahr, M., Allen, L., Magenis, E. Interstitial deletion of (17)(p11.2p11.2) in nine patients. Am. J. Med. Genet. 24: 393-414, 1986.

6. Jary L, Mossafa H, Fourcade C, Genet P, Pulik M, Flandrin $G$ The 17p-syndrome: a distinct myelodysplastic syndrome entity? Leuk Lymphoma. 1997 Mar;25(1-2):163-8.

7. Patil SR, Bartley JA. Interstitial deletion of the short arm of chromosome 17. Hum Genet. 1984; 67(2):237-8.

8. Alice Poisson, Alain Nicolas, Pierre Cochat, Damien Sanlaville, Caroline Rigard, Hélène de Leersnyder, Patricia Franco, Vincent Des Portes, Patrick Edery, Caroline Demily Behavioral disturbance and treatment strategies in SmithMagenis síndrome Orphanet Journal of Rare Diseases (2015) 10:111

9. Smith ACM, McGavran L, Waldstein G. Deletion of the 17 short arm in two patients with facial clefts and congenital heart disease. Am J Hum Genet Suppl 1982;34:A410.

10. Gropman AL, Duncan WC, Smith AC. Neurologic and Developmental Features of the Smith-Magenis Syndrome (del 17p11.2). Pediatry Neurol 2005;34:337-350.

11. Shelley BP, Robertson MM. The Neuropsychiatry and Multisystem Features of the Smith-Magenis Syndrome: A Review. Journal Neuropsychiatry Clin Neurosci 2005; 17:91-97.

12. A Moncla, M O Livet, M Auger, J F Mattei, M G Mattei, and F Giraud. Smith-Magenis syndrome: a new contiguous gene syndrome. Report of three new cases. J Med Genet. 1991 Sep; 28(9): 627-632.

13. Greenberg F, Lewis RA, Potocki L, Glaze D, Parke J, Killian J, Murphy MA, Williamson D, Brown F, Dutton R, McCluggage C, Friedman E, Sulek M, Lupski JR. Multi-disciplinary clinical study of Smith-Magenis syndrome (deletion 17p11.2) Am J Med Genet. 1996 Mar 29;62(3):247-54.

14. Potocki, L., Glaze, D., Tan, D.-X., Park, S.-S., Kashork, C. D., Shaffer, L. G., Reiter, R. J., Lupski, J. R. Circadian rhythm abnormalities of melatonin in Smith-Magenis syndrome. J. Med. Genet. 37: 428-433, 2000
15. De Leersnyder, H., de Blois, M.-C., Claustrat, B., Romana, S., Albrecht, U., von Kleist-Retzow, J.-C., Delobel, B., Viot, G., Lyonnet, S., Vekemans, M., Munnich, A. Inversion of the circadian rhythm of melatonin in the Smith-Magenis syndrome. J. Pediat. 139: 111-116, 2001.

16. Smith, A. C. M., Gropman, A. L., Bailey-Wilson, J. E., GokerAlpan, O., Elsea, S. H., Blancato, J., Lupski, J. R., Potocki, L. Hypercholesterolemia in children with Smith-Magenis syndrome: del (17)(p11.2p11.2). Genet. Med. 4: 118-125, 2002

17. Moncla, A., Piras, L., Arbex, O. F., Muscatelli, F., Mattei, M.-G., Mattei, J.-F., Fontes, M. Physical mapping of microdeletions of the chromosome 17 short arm associated with SmithMagenis syndrome. Hum. Genet. 90: 657-660, 1993.

18. Juyal, R. C., Greenberg, F., Mengden, G. A., Lupski, J. R., Trask, B. J., van den Engh, G., Lindsay, E. A., Christy, H., Chen, K.-S., Baldini, A., Shaffer, L. G., Patel, P. I. SmithMagenis syndrome deletion: a case with equivocal cytogenetic findings resolved by fluorescence in situ hybridization. Am. J. Med. Genet. 58: 286-291, 1995.

19. Chevillard, C., Le Paslier, D., Passage, E., Ougen, P., Billault, A., Boyer, S., Mazan, S., Bachellerie, J. P., Vignal, A., Cohen, D., Fontes, M. Relationship between Charcot-Marie-Tooth $1 \mathrm{~A}$ and Smith-Magenis regions: snU3 may be a candidate gene for the Smith-Magenis syndrome. Hum. Molec. Genet. 2: 1235-1243, 1993.

20. Koyama, K., Fukushima, Y., Inazawa, J., Tomotsune, D., Takahashi, N., Nakamura, Y. The human homologue of the murine Llglh gene (LLGL) maps within the Smith-Magenis syndrome region in 17p11.2. Cytogenet. Cell Genet. 72: 7882, 1996.

21. Lucas, R. E., Vlangos, C. N., Das, P., Patel, P. I., Elsea, S. $\mathrm{H}$. Genomic organisation of the $\sim 1.5 \mathrm{Mb}$ Smith-Magenis syndrome critical interval: transcription map, genomic contig, and candidate gene analysis. Europ. J. Hum. Genet. 9: 892902, 2001.

22. Slager, R. E., Newton, T. L., Vlangos, C. N., Finucane, B., Elsea, S. H. Mutations in RAl1 associated with Smith-Magenis syndrome. Nature Genet. 33: 466-468, 2003.

23. Kaminsky, E. B., Kaul, V., Paschall, J., Church, D. M., Bunke, B., Kunig, D., Moreno-De-Luca, D., Moreno-De-Luca, A., Mulle, J. G., Warren, S. T., Richard, G., Compton, J. G., and 22 others. An evidence-based approach to establish the functional and clinical significance of copy number variants in intellectual and developmental disabilities. Genet. Med. 13: 777-784, 2011. 